\title{
ANALYSES BY THE DEFENSE WASTE PROCESSING FACILITY LABORATORY OF THORIUM GLASSES FROM THE SLUDGE BATCH 6 VARIABILITY STUDY
}

\author{
M.T. Feller \\ D.R. Click \\ T.B. Edwards
}

February 2011

Applied Computational Engineering and Statistics Savannah River National Laboratory

Aiken, SC 29808

This document was prepared in conjunction with work accomplished under Contract No. DE-AC09-08SR22470 with the U.S. Department of Energy.

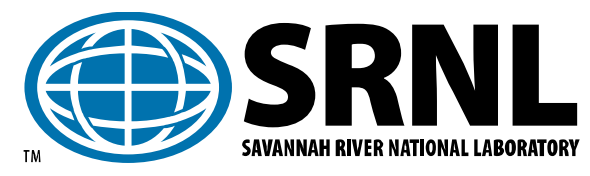


SRNL-STI-2011-00074

Revision 0

\section{DISCLAIMER}

This work was prepared under an agreement with and funded by the U.S. Government. Neither the U.S. Government or its employees, nor any of its contractors, subcontractors or their employees, makes any express or implied: 1 . warranty or assumes any legal liability for the accuracy, completeness, or for the use or results of such use of any information, product, or process disclosed; or 2. representation that such use or results of such use would not infringe privately owned rights; or 3. endorsement or recommendation of any specifically identified commercial product, process, or service. Any views and opinions of authors expressed in this work do not necessarily state or reflect those of the United States Government, or its contractors, or subcontractors.

This document was prepared in conjunction with work accomplished under Contract No. DE-AC09-08SR22470 with the U.S. Department of Energy. 
SRNL-STI-2011-00074

Revision 0

\section{REVIEWS AND APPROVALS}

\section{AUTHORS:}

D. R. Click, Spectroscopy and Separations

Date

Savannah River National Laboratory

M. T. Feller, Waste Laboratory Services

Date

Savannah River Remediation

T. B. Edwards, Applied Computational Engineering and Statistics Date

Savannah River National Laboratory

TECHNICAL REVIEWERS:

D. R. Best, Process Technology Programs

Date

Savannah River National Laboratory

E. P. Shine, Applied Computational Engineering and Statistics

Date

Savannah River National Laboratory

\section{APPROVERS:}

P. L. Lee, Manager, Applied Computational Engineering and Statistics Date Savannah River National Laboratory

C. C. Herman, Manager, Process Technology Programs

Date Savannah River National Laboratory

R. T. McNew, Manager, Waste Laboratory Services

Date

Savannah River Remediation

J. E. Occhipinti, Manager, Waste Solidification Engineering

Date

Savannah River Remediation 


\section{EXECUTIVE SUMMARY}

The Savannah River Remediation (SRR) Defense Waste Processing Facility (DWPF) is currently processing Sludge Batch 6 (SB6) with Frit 418. At times during the processing of this glass system, thorium is expected to be at concentrations in the final wasteform that make it a reportable element for the first time since startup of radioactive operations at the DWPF. The Savannah River National Laboratory (SRNL) supported the qualification of the processing of this glass system at the DWPF. A recommendation from the SRNL studies was the need for the DWPF Laboratory to establish a method to measure thorium by Inductively Coupled Plasma - Atomic Emission Spectroscopy (ICPAES). This recommendation led to the set of thorium-bearing glasses from the SB6 Variability Study (VS) being submitted to the DWPF Laboratory for chemical composition measurement. The measurements were conducted by the DWPF Laboratory using the sodium peroxide fusion preparation method routinely employed for analysis of samples from the Slurry Mix Evaporator (SME). These measurements are presented and reviewed in this report. The review indicates that the measurements provided by the DWPF Laboratory are comparable to those provided by Analytical Development's laboratory at SRNL for these same glasses. As a result, the authors of this report recommend that the DWPF Laboratory begin using its routine peroxide fusion dissolution method for the measurement of thorium in SME samples of SB6. 


\section{TABLE OF CONTENTS}

LIST OF ABBREVIATIONS

\section{LIST OF ABBREVIATIONS}

$\begin{array}{ll}\text { AD } & \text { Analytical Development } \\ \text { DWPF } & \text { Defense Waste Processing Facility } \\ \text { ICP-AES } & \text { Inductively Coupled Plasma - Atomic Emission Spectroscopy } \\ \text { JMP } & \text { Statistical software package from SAS Institute, Inc. [3] } \\ \text { SB6 } & \text { Sludge Batch 6 } \\ \text { SB6 VS } & \text { Sludge Batch 6 Variability Study } \\ \text { SME } & \text { Slurry Mix Evaporator } \\ \text { SRNL } & \text { Savannah River National Laboratory } \\ \text { VS } & \text { Variability Study } \\ \text { wt } \% & \text { Weight percent }\end{array}$




\subsection{INTRODUCTION}

The Savannah River Remediation (SRR) Defense Waste Processing Facility (DWPF) is currently processing Sludge Batch 6 (SB6) with Frit 418. At times during the processing of this glass system, thorium is expected to be at concentrations in the final wasteform that make it a reportable element for the first time since the startup of radioactive operations at the DWPF. The Savannah River National Laboratory (SRNL) supported the qualification of the processing of this glass system at the DWPF. A recommendation from one of the supporting studies [1] was the need for the DWPF Laboratory to establish a method to measure thorium by Inductively Coupled Plasma - Atomic Emission Spectroscopy (ICP-AES). This recommendation led to the set of thorium-bearing glasses from the SB6 Variability Study (VS) [2] being submitted to the DWPF Laboratory for measurement of their chemical compositions. The measurements were conducted by the DWPF Laboratory using the sodium peroxide fusion preparation method routinely employed for analysis of samples from the Slurry Mix Evaporator (SME). The use of this preparation method for these analyses was guided by information regarding analytical issues, outlined in [2], that are associated with thorium being present in these glasses.

The purpose of this technical report is to present the measurements generated by the DWPF Laboratory for the SB6 VS glasses and to compare the measurements to the targeted compositions for these VS glasses as well as to SRNL's measurements (both sets, targeted and measured, of compositional values were reported by SRNL in [2]). The goal of these comparisons is to provide information that will lead to the qualification of peroxide fusion dissolution as a method for the measurement by the DWPF Laboratory of thorium in SME glass samples.

\subsection{ANALYSES AND COMPARISONS}

Five glasses of the SB6 VS were batched to contain reportable quantities of thorium (i.e., elemental concentrations of thorium greater than 0.5 weight percent (wt\%) of the glass) [2]. The identifying labels of these glasses were SB6VS-18, SB6VS-19, SB6VS-20, SB6VS-21, and SV6VS-22, and these glasses were selected to pursue the implementation of the recommendation cited above regarding the need to establish at DWPF Laboratory a method to measure thorium by ICP-AES.

\subsection{Measurements by DWPF Laboratory}

Samples of these five glasses were transferred from SRNL to the DWPF Laboratory. The samples were analyzed by peroxide fusion dissolution and the resulting measurements are presented in Table A1 through Table A5 of the Appendix. Note that no sodium or zirconium values are provided in these tables. Measurements of these elements are not available from samples prepared by the peroxide fusion method. Also, note that copper and titanium concentrations were not provided for all of the glasses. Since these two elements are present only in small quantities in all of these glasses, these missing values do not affect the conclusions of this report. The elemental concentrations were converted to oxide concentrations by multiplying by the appropriate gravimetric factors. For those results with duplicate measurements, the average value was determined. The resulting values are presented in Table 1. 
Table 1. Measured Oxide Concentrations (wt\%’s) by DWPF Laboratory

\begin{tabular}{|l|r|r|r|r|r|}
\hline & \multicolumn{5}{|c|}{ Glass ID } \\
\cline { 2 - 6 } Oxide & \multicolumn{6}{|c|}{ SB6VS18 } & \multicolumn{1}{c|}{ SB6VS19 } & SB6VS20 & SB6VS21 & SB6VS22 \\
\hline $\mathrm{Al}_{2} \mathrm{O}_{3}$ & 8.267 & 8.797 & 9.474 & 9.755 & 10.251 \\
\hline $\mathrm{B}_{2} \mathrm{O}_{3}$ & 5.173 & 4.979 & 4.785 & 4.588 & 4.469 \\
\hline $\mathrm{CaO}$ & 0.378 & 0.423 & 0.497 & 0.569 & 0.558 \\
\hline $\mathrm{Cr}_{2} \mathrm{O}_{3}$ & 0.049 & 0.062 & 0.028 & 0.020 & 0.034 \\
\hline $\mathrm{CuO}$ & Not reported & Not reported & 0.038 & 0.034 & 0.041 \\
\hline $\mathrm{Fe}_{2} \mathrm{O}_{3}$ & 7.537 & 7.803 & 8.226 & 8.494 & 9.021 \\
\hline $\mathrm{K}_{2} \mathrm{O}$ & 0.648 & 1.010 & 0.025 & 0.049 & 0.108 \\
\hline $\mathrm{Li} 2 \mathrm{O}$ & 5.406 & 5.217 & 5.169 & 4.904 & 4.902 \\
\hline $\mathrm{MgO}$ & 0.254 & 0.261 & 0.300 & 0.292 & 0.322 \\
\hline $\mathrm{MnO}$ & 2.137 & 2.238 & 2.309 & 2.403 & 2.571 \\
\hline $\mathrm{NiO}^{2} \mathrm{O}$ & 0.906 & 0.990 & 0.942 & 0.962 & 1.052 \\
\hline $\mathrm{SiO}_{2}$ & 52.163 & 48.641 & 50.066 & 47.569 & 46.213 \\
\hline $\mathrm{ThO}_{2}$ & 1.041 & 1.101 & 1.084 & 1.123 & 1.199 \\
\hline $\mathrm{TiO}_{2}$ & Not reported & Not reported & 0.342 & 0.330 & 0.369 \\
\hline $\mathrm{U}_{3} \mathrm{O}_{8}$ & 1.456 & 1.688 & 1.404 & 1.432 & 1.579 \\
\hline
\end{tabular}

\subsection{Tabulated Comparisons of Measured versus Targeted Oxide Concentrations}

Since these thorium glasses that were analyzed by the DWPF Laboratory were part of the SB6 VS conducted by SRNL, their targeted concentrations and the results of their compositional measurements by SRNL's Analytical Development (AD) laboratory are available in the SB6 VS report [2]. Table 2 provides the results from the SB6 VS along with the DWPF Laboratories' measurements.

Table 2. Targeted and Measured Oxide Concentrations for the Thorium Glasses

\begin{tabular}{|c|c|c|c|c|c|c|c|c|}
\hline & & & \multicolumn{3}{|c|}{ SRNL Laboratory } & \multicolumn{3}{|c|}{ DWPF Laboratory } \\
\hline & & Targeted & Measured & Diff. of & \% Diff. of & Measured & Diff. of & \% Diff. of \\
\hline Glass ID & Oxide & (wt\%) & (wt\%) & Measured & Measured & Oxide wt \% & Measured & Measured \\
\hline SB6VS18 & $\mathrm{Al}_{2} \mathrm{O}_{3}$ & 8.1160 & 8.4933 & 0.3773 & $4.6 \%$ & 8.267 & 0.1511 & $1.9 \%$ \\
\hline SB6VS18 & $\mathrm{B}_{2} \mathrm{O}_{3}$ & 5.4400 & 5.2565 & -0.1835 & $-3.4 \%$ & 5.173 & -0.2669 & $-4.9 \%$ \\
\hline SB6VS18 & $\mathrm{CaO}$ & 0.4390 & 0.4334 & -0.0056 & $-1.3 \%$ & 0.378 & -0.0607 & $-13.8 \%$ \\
\hline SB6VS18 & $\mathrm{Cr}_{2} \mathrm{O}_{3}$ & 0.0380 & 0.0333 & -0.0047 & $-12.4 \%$ & 0.049 & 0.0111 & $29.2 \%$ \\
\hline SB6VS18 & $\mathrm{CuO}$ & 0.0330 & 0.0338 & 0.0008 & $2.4 \%$ & \multicolumn{3}{|c|}{ Not Available } \\
\hline SB6VS18 & $\mathrm{Fe}_{2} \mathrm{O}_{3}$ & 7.5090 & 7.3773 & -0.1317 & $-1.8 \%$ & 7.537 & 0.0282 & $0.4 \%$ \\
\hline SB6VS18 & $\mathrm{K}_{2} \mathrm{O}$ & 0.0270 & 0.1622 & 0.1352 & $500.6 \%$ & 0.648 & 0.6206 & $2298.5 \%$ \\
\hline SB6VS18 & $\mathrm{Li}_{2} \mathrm{O}$ & 5.4400 & 5.3069 & -0.1331 & $-2.4 \%$ & 5.406 & -0.0343 & $-0.6 \%$ \\
\hline SB6VS18 & $\mathrm{MgO}$ & 0.2440 & 0.2442 & 0.0002 & $0.1 \%$ & 0.254 & 0.0102 & $4.2 \%$ \\
\hline SB6VS18 & $\mathrm{MnO}$ & 2.1370 & 2.0885 & -0.0485 & $-2.3 \%$ & 2.137 & -0.0005 & $0.0 \%$ \\
\hline SB6VS18 & $\mathrm{NiO}$ & 0.9160 & 0.8440 & -0.0720 & $-7.9 \%$ & 0.906 & -0.0105 & $-1.1 \%$ \\
\hline SB6VS18 & $\mathrm{SiO}_{2}$ & 52.3530 & 54.0708 & 1.7178 & $3.3 \%$ & 52.163 & -0.1898 & $-0.4 \%$ \\
\hline SB6VS18 & $\mathrm{ThO}_{2}$ & 1.0110 & 0.8739 & -0.1371 & $-13.6 \%$ & 1.041 & 0.0304 & $3.0 \%$ \\
\hline SB6VS18 & $\mathrm{TiO}_{2}$ & 0.2940 & 0.3002 & 0.0062 & $2.1 \%$ & \multicolumn{3}{|c|}{ Not Available } \\
\hline SB6VS18 & $\mathrm{U}_{3} \mathrm{O}_{8}$ & 1.4980 & 1.4298 & -0.0682 & $-4.6 \%$ & 1.456 & -0.0423 & $-2.8 \%$ \\
\hline
\end{tabular}


Table 2. Targeted and Measured Oxide Concentrations for the Thorium Glasses (continued)

\begin{tabular}{|c|c|c|c|c|c|c|c|c|}
\hline & & & \multicolumn{3}{|c|}{ SRNL Laboratory } & \multicolumn{3}{|c|}{ DWPF Laboratory } \\
\hline & & Targeted & Measured & Diff. of & \% Diff. of & Measured & Diff. of & \% Diff. of \\
\hline Glass ID & Oxide & (wt\%) & (wt\%) & Measured & Measured & Oxide wt $\%$ & Measured & Measured \\
\hline SB6VS19 & $\mathrm{Al}_{2} \mathrm{O}_{3}$ & 8.6230 & 9.0224 & 0.3994 & $4.6 \%$ & 8.797 & 0.1741 & $2.0 \%$ \\
\hline SB6VS19 & $\mathrm{B}_{2} \mathrm{O}_{3}$ & 5.2800 & 5.0794 & -0.2006 & $-3.8 \%$ & 4.979 & -0.3011 & $-5.7 \%$ \\
\hline SB6VS19 & $\mathrm{CaO}$ & 0.4670 & 0.4866 & 0.0196 & $4.2 \%$ & 0.423 & -0.0440 & $-9.4 \%$ \\
\hline SB6VS19 & $\mathrm{Cr}_{2} \mathrm{O}_{3}$ & 0.0400 & 0.0377 & -0.0023 & $-5.8 \%$ & 0.062 & 0.0217 & $54.2 \%$ \\
\hline SB6VS19 & $\mathrm{CuO}$ & 0.0360 & 0.0351 & -0.0009 & $-2.4 \%$ & \multicolumn{3}{|c|}{ Not Available } \\
\hline SB6VS19 & $\mathrm{Fe}_{2} \mathrm{O}_{3}$ & 7.9780 & 7.8026 & -0.1754 & $-2.2 \%$ & 7.803 & -0.1747 & $-2.2 \%$ \\
\hline SB6VS19 & $\mathrm{K}_{2} \mathrm{O}$ & 0.0290 & 0.2417 & 0.2127 & $733.4 \%$ & 1.010 & 0.9812 & $3383.4 \%$ \\
\hline SB6VS19 & $\mathrm{Li}_{2} \mathrm{O}$ & 5.2800 & 5.1562 & -0.1238 & $-2.3 \%$ & 5.217 & -0.0631 & $-1.2 \%$ \\
\hline SB6VS19 & $\mathrm{MgO}$ & 0.2590 & 0.2554 & -0.0036 & $-1.4 \%$ & 0.261 & 0.0017 & $0.7 \%$ \\
\hline SB6VS19 & $\mathrm{MnO}$ & 2.2700 & 2.2209 & -0.0491 & $-2.2 \%$ & 2.238 & -0.0321 & $-1.4 \%$ \\
\hline SB6VS19 & $\mathrm{NiO}$ & 0.9730 & 0.8917 & -0.0813 & $-8.4 \%$ & 0.990 & 0.0174 & $1.8 \%$ \\
\hline SB6VS19 & $\mathrm{SiO}_{2}$ & 50.8750 & 52.6803 & 1.8053 & $3.5 \%$ & 48.641 & -2.2337 & $-4.4 \%$ \\
\hline SB6VS19 & $\mathrm{ThO}_{2}$ & 1.0740 & 0.9123 & -0.1617 & $-15.1 \%$ & 1.101 & 0.0266 & $2.5 \%$ \\
\hline SB6VS19 & $\mathrm{TiO}_{2}$ & 0.3130 & 0.3186 & 0.0056 & $1.8 \%$ & \multicolumn{3}{|c|}{ Not Available } \\
\hline SB6VS19 & $\mathrm{U}_{3} \mathrm{O}_{8}$ & 1.5910 & 1.4504 & -0.1406 & $-8.8 \%$ & 1.688 & 0.0968 & $6.1 \%$ \\
\hline SB6VS20 & $\mathrm{Al}_{2} \mathrm{O}_{3}$ & 9.1300 & 9.5231 & 0.3931 & $4.3 \%$ & 9.474 & 0.3440 & $3.8 \%$ \\
\hline SB6VS20 & $\mathrm{B}_{2} \mathrm{O}_{3}$ & 5.1200 & 4.9264 & -0.1936 & $-3.8 \%$ & 4.785 & -0.3352 & $-6.5 \%$ \\
\hline SB6VS20 & $\mathrm{CaO}$ & 0.4940 & 0.4656 & -0.0284 & $-5.8 \%$ & 0.497 & 0.0027 & $0.5 \%$ \\
\hline SB6VS20 & $\mathrm{Cr}_{2} \mathrm{O}_{3}$ & 0.0430 & 0.0369 & -0.0061 & $-14.3 \%$ & 0.028 & -0.0152 & $-35.4 \%$ \\
\hline SB6VS20 & $\mathrm{CuO}$ & 0.0380 & 0.0367 & -0.0013 & $-3.4 \%$ & 0.038 & -0.0004 & $-1.2 \%$ \\
\hline SB6VS20 & $\mathrm{Fe}_{2} \mathrm{O}_{3}$ & 8.4470 & 8.2458 & -0.2012 & $-2.4 \%$ & 8.226 & -0.2205 & $-2.6 \%$ \\
\hline SB6VS20 & $\mathrm{K}_{2} \mathrm{O}$ & 0.0300 & 0.3426 & 0.3126 & $1041.9 \%$ & 0.025 & -0.0047 & $-15.7 \%$ \\
\hline SB6VS20 & $\mathrm{Li}_{2} \mathrm{O}$ & 5.1200 & 5.0163 & -0.1037 & $-2.0 \%$ & 5.169 & 0.0491 & $1.0 \%$ \\
\hline SB6VS20 & $\mathrm{MgO}$ & 0.2750 & 0.2699 & -0.0051 & $-1.9 \%$ & 0.300 & 0.0252 & $9.1 \%$ \\
\hline SB6VS20 & $\mathrm{MnO}$ & 2.4040 & 2.3274 & $\begin{array}{l}-0.0766 \\
\end{array}$ & $-3.2 \%$ & 2.309 & -0.0953 & $-4.0 \%$ \\
\hline SB6VS20 & $\mathrm{NiO}$ & 1.0300 & 0.9251 & -0.1049 & $-10.2 \%$ & 0.942 & -0.0884 & $-8.6 \%$ \\
\hline SB6VS20 & $\mathrm{SiO}_{2}$ & 49.3970 & 50.7549 & 1.3579 & $2.7 \%$ & 50.066 & 0.6690 & $1.4 \%$ \\
\hline SB6VS20 & $\mathrm{ThO}_{2}$ & 1.1370 & 0.9814 & -0.1556 & $-13.7 \%$ & 1.084 & -0.0526 & $-4.6 \%$ \\
\hline SB6VS20 & $\mathrm{TiO}_{2}$ & 0.3310 & 0.3319 & 0.0009 & $0.3 \%$ & 0.342 & 0.0109 & $3.3 \%$ \\
\hline SB6VS20 & $\mathrm{U}_{3} \mathrm{O}_{8}$ & 1.6850 & 1.6037 & -0.0813 & $-4.8 \%$ & 1.404 & -0.2806 & $-16.7 \%$ \\
\hline
\end{tabular}


Table 2. Targeted and Measured Oxide Concentrations for the Thorium Glasses (continued)

\begin{tabular}{|c|c|c|c|c|c|c|c|c|}
\hline & & & \multicolumn{3}{|c|}{ SRNL Laboratory } & \multicolumn{3}{|c|}{ DWPF Laboratory } \\
\hline & & Targeted & Measured & Diff. of & \% Diff. of & Measured & Diff. of & \% Diff. of \\
\hline Glass ID & Oxide & (wt\%) & (wt\%) & Measured & Measured & Oxide wt $\%$ & Measured & Measured \\
\hline SB6VS21 & $\mathrm{Al}_{2} \mathrm{O}_{3}$ & 9.6370 & 10.1277 & 0.4907 & $5.1 \%$ & 9.755 & 0.1185 & $1.2 \%$ \\
\hline SB6VS21 & $\mathrm{B}_{2} \mathrm{O}_{3}$ & 4.9600 & 4.7494 & -0.2106 & $-4.2 \%$ & 4.588 & -0.3716 & $-7.5 \%$ \\
\hline SB6VS21 & $\mathrm{CaO}$ & 0.5220 & 0.5523 & 0.0303 & $5.8 \%$ & 0.569 & 0.0475 & $9.1 \%$ \\
\hline SB6VS21 & $\mathrm{Cr}_{2} \mathrm{O}_{3}$ & 0.0450 & 0.0365 & -0.0085 & $-19.0 \%$ & 0.020 & -0.0245 & $-54.5 \%$ \\
\hline SB6VS21 & $\mathrm{CuO}$ & 0.0400 & 0.0398 & -0.0002 & $-0.6 \%$ & 0.034 & -0.0062 & $-15.5 \%$ \\
\hline SB6VS21 & $\mathrm{Fe}_{2} \mathrm{O}_{3}$ & 8.9170 & 8.7462 & -0.1708 & $-1.9 \%$ & 8.494 & -0.4232 & $-4.7 \%$ \\
\hline SB6VS21 & $\mathrm{K}_{2} \mathrm{O}$ & 0.0320 & 0.3099 & 0.2779 & $868.4 \%$ & 0.049 & 0.0174 & $54.3 \%$ \\
\hline SB6VS21 & $\mathrm{Li}_{2} \mathrm{O}$ & 4.9600 & 4.8548 & -0.1052 & $-2.1 \%$ & 4.904 & -0.0557 & $-1.1 \%$ \\
\hline SB6VS21 & $\mathrm{MgO}$ & 0.2900 & 0.2861 & -0.0039 & $-1.4 \%$ & 0.292 & 0.0019 & $0.6 \%$ \\
\hline SB6VS21 & $\mathrm{MnO}$ & 2.5370 & 2.4565 & -0.0805 & $-3.2 \%$ & 2.403 & -0.1341 & $-5.3 \%$ \\
\hline SB6VS21 & $\mathrm{NiO}$ & 1.0880 & 0.9849 & -0.1031 & $-9.5 \%$ & 0.962 & -0.1260 & $-11.6 \%$ \\
\hline SB6VS21 & $\mathrm{SiO}_{2}$ & 47.9190 & 49.5783 & 1.6593 & $3.5 \%$ & 47.569 & -0.3495 & $-0.7 \%$ \\
\hline SB6VS21 & $\mathrm{ThO}_{2}$ & 1.2000 & 1.0662 & -0.1338 & $-11.1 \%$ & 1.123 & -0.0769 & $-6.4 \%$ \\
\hline SB6VS21 & $\mathrm{TiO}_{2}$ & 0.3500 & 0.3494 & -0.0006 & $-0.2 \%$ & 0.330 & -0.0197 & $-5.6 \%$ \\
\hline SB6VS21 & $\mathrm{U}_{3} \mathrm{O}_{8}$ & 1.7780 & 1.7511 & -0.0269 & $-1.5 \%$ & 1.432 & -0.3465 & $-19.5 \%$ \\
\hline SB6VS22 & $\mathrm{Al}_{2} \mathrm{O}_{3}$ & 10.1450 & 10.5245 & 0.3795 & $3.7 \%$ & 10.251 & 0.1055 & $1.0 \%$ \\
\hline SB6VS22 & $\mathrm{B}_{2} \mathrm{O}_{3}$ & 4.8000 & 4.6206 & -0.1794 & $-3.7 \%$ & 4.469 & -0.3308 & $-6.9 \%$ \\
\hline SB6VS22 & $\mathrm{CaO}$ & 0.5490 & 0.5058 & -0.0432 & $-7.9 \%$ & 0.558 & 0.0093 & $1.7 \%$ \\
\hline SB6VS22 & $\mathrm{Cr}_{2} \mathrm{O}_{3}$ & 0.0470 & 0.0429 & -0.0041 & $-8.7 \%$ & 0.034 & -0.0134 & $-28.5 \%$ \\
\hline SB6VS22 & $\mathrm{CuO}$ & 0.0420 & 0.0406 & -0.0014 & $-3.3 \%$ & 0.041 & -0.0007 & $-1.6 \%$ \\
\hline SB6VS22 & $\mathrm{Fe}_{2} \mathrm{O}_{3}$ & 9.3860 & 9.1965 & -0.1895 & $-2.0 \%$ & 9.021 & -0.3646 & $-3.9 \%$ \\
\hline SB6VS22 & $\mathrm{K}_{2} \mathrm{O}$ & 0.0340 & 0.2259 & 0.1919 & $564.3 \%$ & 0.108 & 0.0744 & $218.9 \%$ \\
\hline SB6VS22 & $\mathrm{Li}_{2} \mathrm{O}$ & 4.8000 & 4.7149 & -0.0851 & $-1.8 \%$ & 4.902 & 0.1022 & $2.1 \%$ \\
\hline SB6VS22 & $\mathrm{MgO}$ & 0.3050 & 0.3031 & -0.0019 & $-0.6 \%$ & 0.322 & 0.0167 & $5.5 \%$ \\
\hline SB6VS22 & $\mathrm{MnO}$ & 2.6710 & 2.6276 & -0.0434 & $-1.6 \%$ & 2.571 & $\begin{array}{l}-0.1002 \\
\end{array}$ & $-3.8 \%$ \\
\hline SB6VS22 & $\mathrm{NiO}$ & 1.1450 & 1.0390 & -0.1060 & $-9.3 \%$ & 1.052 & -0.0926 & $-8.1 \%$ \\
\hline SB6VS22 & $\mathrm{SiO}_{2}$ & 46.4410 & 47.9203 & 1.4793 & $3.2 \%$ & 46.213 & -0.2278 & $-0.5 \%$ \\
\hline SB6VS22 & $\mathrm{ThO}_{2}$ & 1.2630 & 1.1248 & -0.1382 & $-10.9 \%$ & 1.199 & -0.0637 & $-5.0 \%$ \\
\hline SB6VS22 & $\mathrm{TiO}_{2}$ & 0.3680 & 0.3715 & 0.0035 & $1.0 \%$ & 0.369 & 0.0006 & $0.2 \%$ \\
\hline SB6VS22 & $\mathrm{U}_{3} \mathrm{O}_{8}$ & 1.8720 & 1.8130 & -0.0590 & $-3.2 \%$ & 1.579 & -0.2931 & $-15.7 \%$ \\
\hline
\end{tabular}

In addition to the targeted and measured concentrations, the differences between the measured and targeted concentrations are provided for both laboratories as well as the percent differences relative to the targeted concentrations for both laboratories. The targeted concentrations are used as the points of comparison since there were no issues seen in the batching of these VS glasses [2]. While there are large percent differences for those oxides at smaller concentrations in these glasses for both laboratories, the entries in this table suggest comparable results for the two laboratories for the measurement of these glasses.

One difference that should be noted between the performance of the fusion methods is that the DWPF Laboratory uses $50 \mathrm{~mL}$ of $1: 1 \mathrm{HCl}$ to $\mathrm{H}_{2} \mathrm{O}$ uptake for the fusion whereas SRNL uses $25 \mathrm{~mL}$ of concentrated $\mathrm{HNO}_{3}$ and sometimes adds $\mathrm{H}_{2} \mathrm{O}_{2}$ to clarify the solutions. Both laboratories finish volumetric dilution of the samples using deionized water. 


\subsection{Supporting Plots Comparing the Results}

While the information of Table 1 provides an opportunity for a close comparison of the results from the two laboratories for each glass, the plots of Exhibit A1 in the Appendix allow for a comparison of the targeted, SRNL measured, and DWPF measured values for each oxide for all of the glasses. The plots were developed using JMP Version 7.0.2 [3], and they illustrate the performance of the DWPF Laboratory relative to that of the $\mathrm{AD}$ laboratory in measuring the oxide concentrations of these thorium glasses. Once again, there is no indication of a problem seen in these plots. Specifically, the DWPF Laboratory measurements for $\mathrm{ThO}_{2}$ fall closer to the targeted values than do the AD values.

\subsection{CONCLUSIONS AND RECOMMENDATIONS}

The measurements generated by the DWPF Laboratory for the thorium glasses from SRNL's SB6 VS are presented and reviewed in this report. The DWPF Laboratory used the peroxide fusion dissolution method that it routinely employs in the ICP-AES analysis of SME samples to conduct these measurements. The review of these measurements that is provided in this report indicates that the measurements provided by the DWPF Laboratory are comparable to those provided by AD's laboratory for these same glasses. As a result, the authors of this report recommend that the DWPF Laboratory begin using its routine peroxide fusion dissolution method for the measurement of thorium in SME samples of SB6.

\section{REFERENCES}

[1] Click, D.R., T.B. Edwards, M.A. Jones, "Verification of the Defense Waste Processing Facility’s (DWPF) Process Digestion Method for the Sludge Batch 6 Qualification Sample,” SRNL-STI-2010-00259, Revision 0, June 2010.

[2] Johnson, F.C. and T.B. Edwards, “Sludge Batch 6 Variability Study with Frit 418,” SRNLSTI-2010-00242, Revision 0, November 2010.

[3] JMP Version 7.0.2, SAS Institute, Inc., Cary NC, 1989-2007. 
SRNL-STI-2011-00074

Revision 0

\section{APPENDIX}

Table A1. DWPF Laboratory Measurements of SB6VS-18

DWPF_RSD_report

Report Date 14-DEC-10 02:14 PM

Sample Id 200004122

Logged 13-DEC-10

Sample Type SME-1

Condition ONLINE

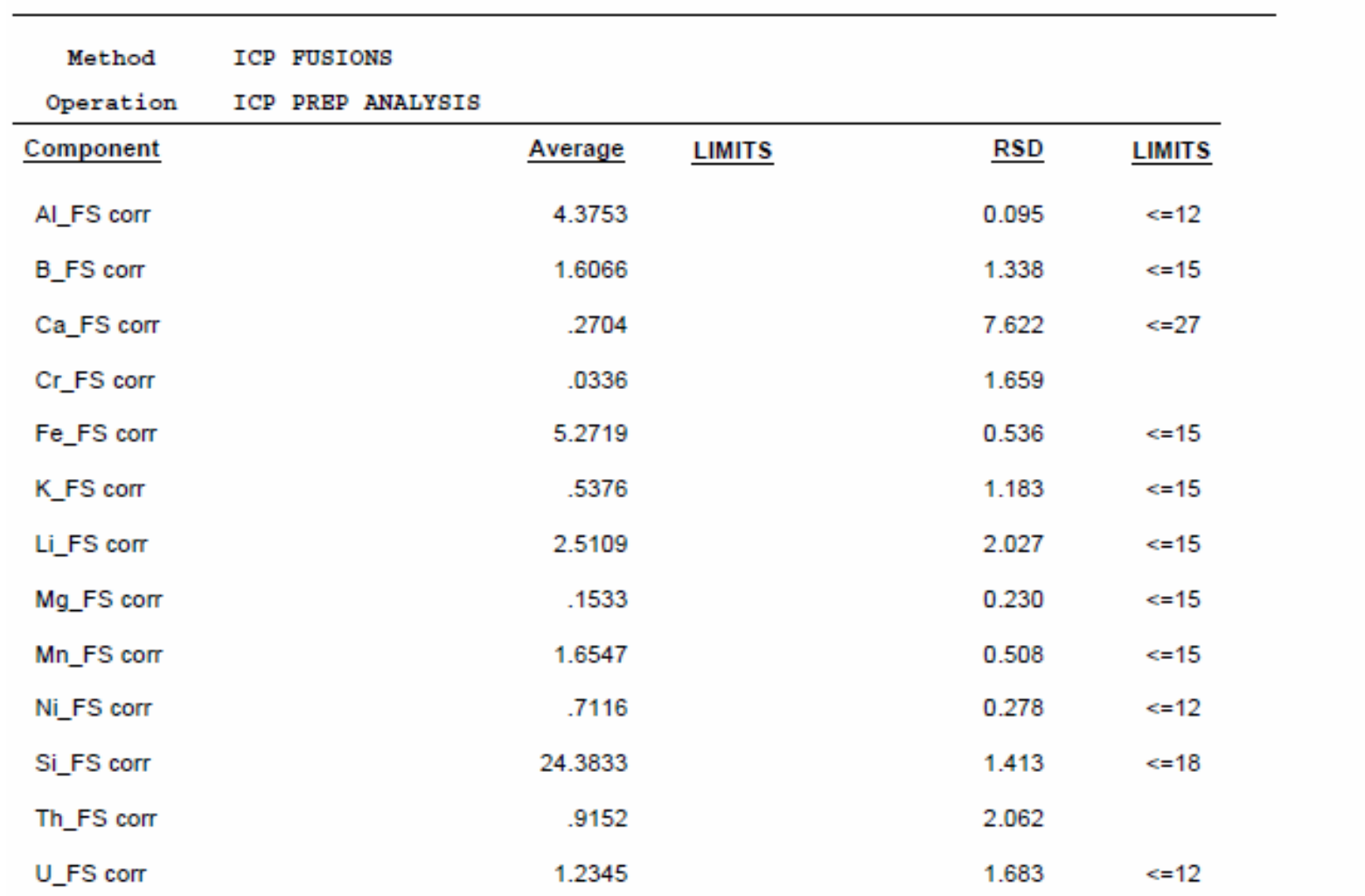


SRNL-STI-2011-00074

Revision 0

Table A2. DWPF Laboratory Measurements of SB6VS-19

DWPF_RSD_report

Sample Id 200004123

Sample Type SME-1
Report Date 14-DEC-10 02:19 PM

Logged 13-DEC-10

Logged by L4097

Condition ONLINE

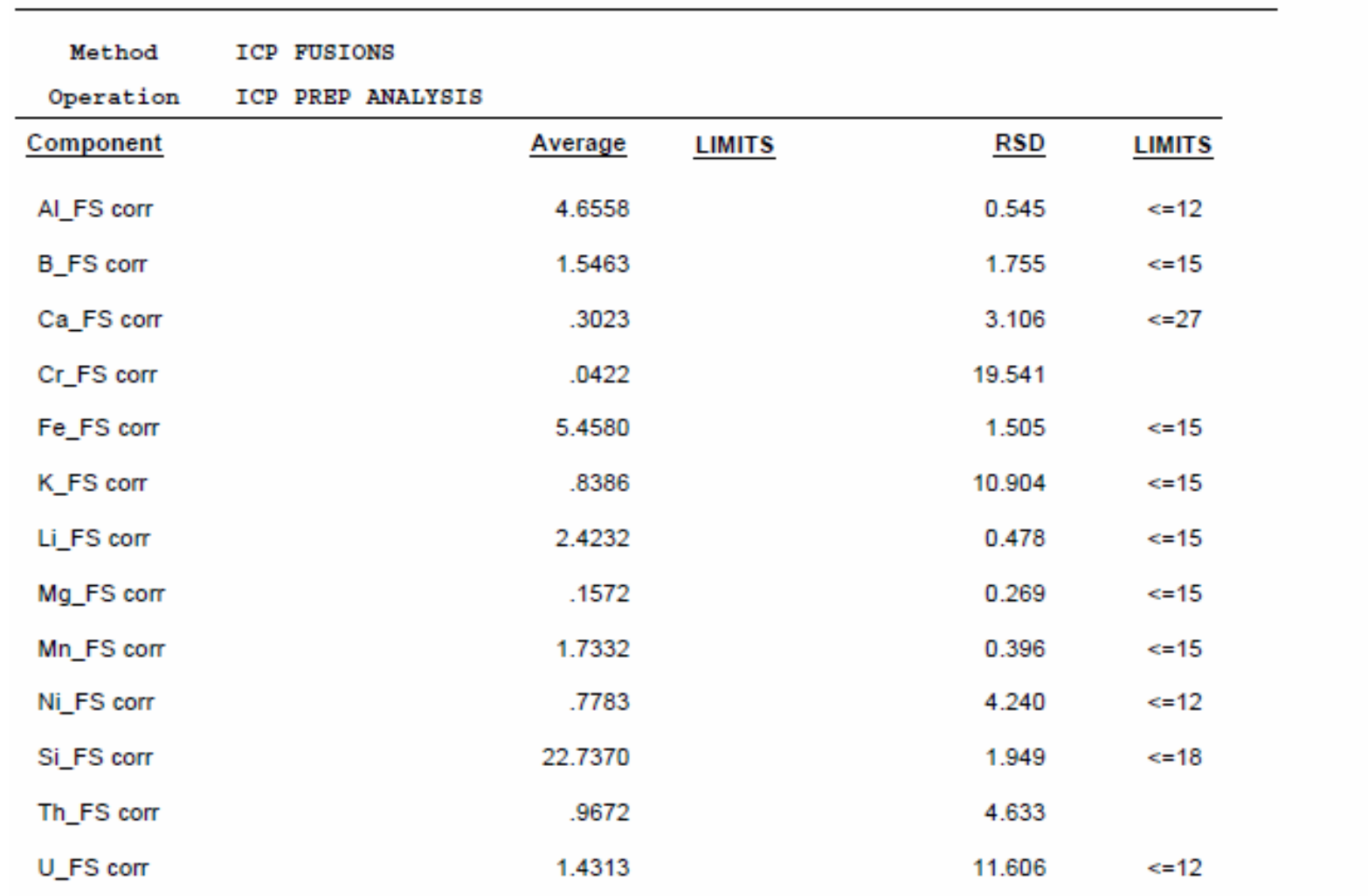


SRNL-STI-2011-00074

Revision 0

Table A3. DWPF Laboratory Measurements of SB6VS-20

Analytical Report

DWPF_sample_report
DWPF 221-S Laboratory
200003268

Page 1 of 2

Report Date $\quad$ 26-OCT-10 05:03 AM

Submission ID 100003268

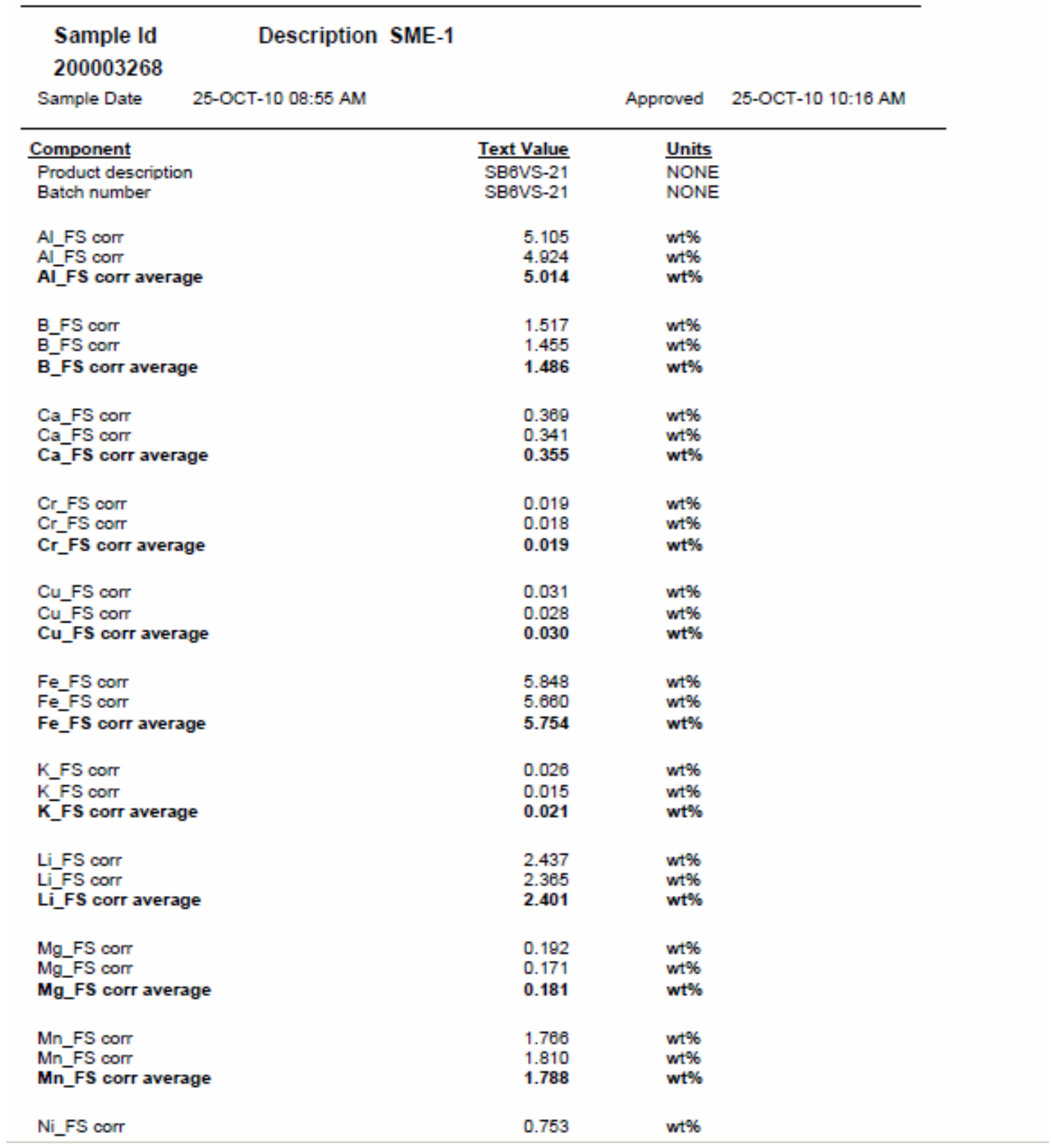


SRNL-STI-2011-00074

Revision 0

Table A3. DWPF Laboratory Measurements of SB6VS-20 (continued)

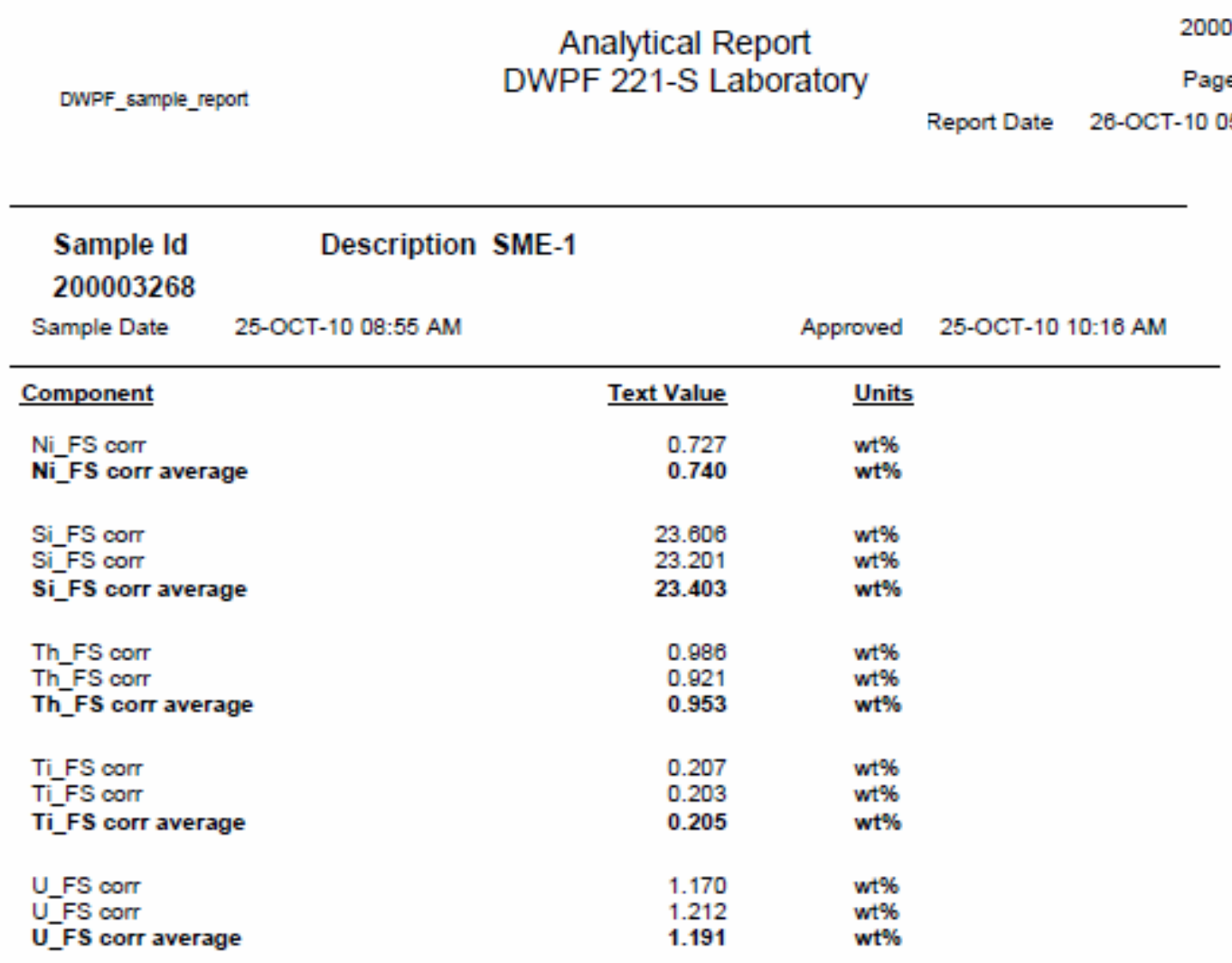


SRNL-STI-2011-00074

Revision 0

Table A4. DWPF Laboratory Measurements of SB6VS-21

Analytical Report

DWPF_sample_report
DWPF 221-S Laboratory
200003269

Page 1 of 2

Report Date 26-OCT-10 05:04 AM

Submission ID 100003269

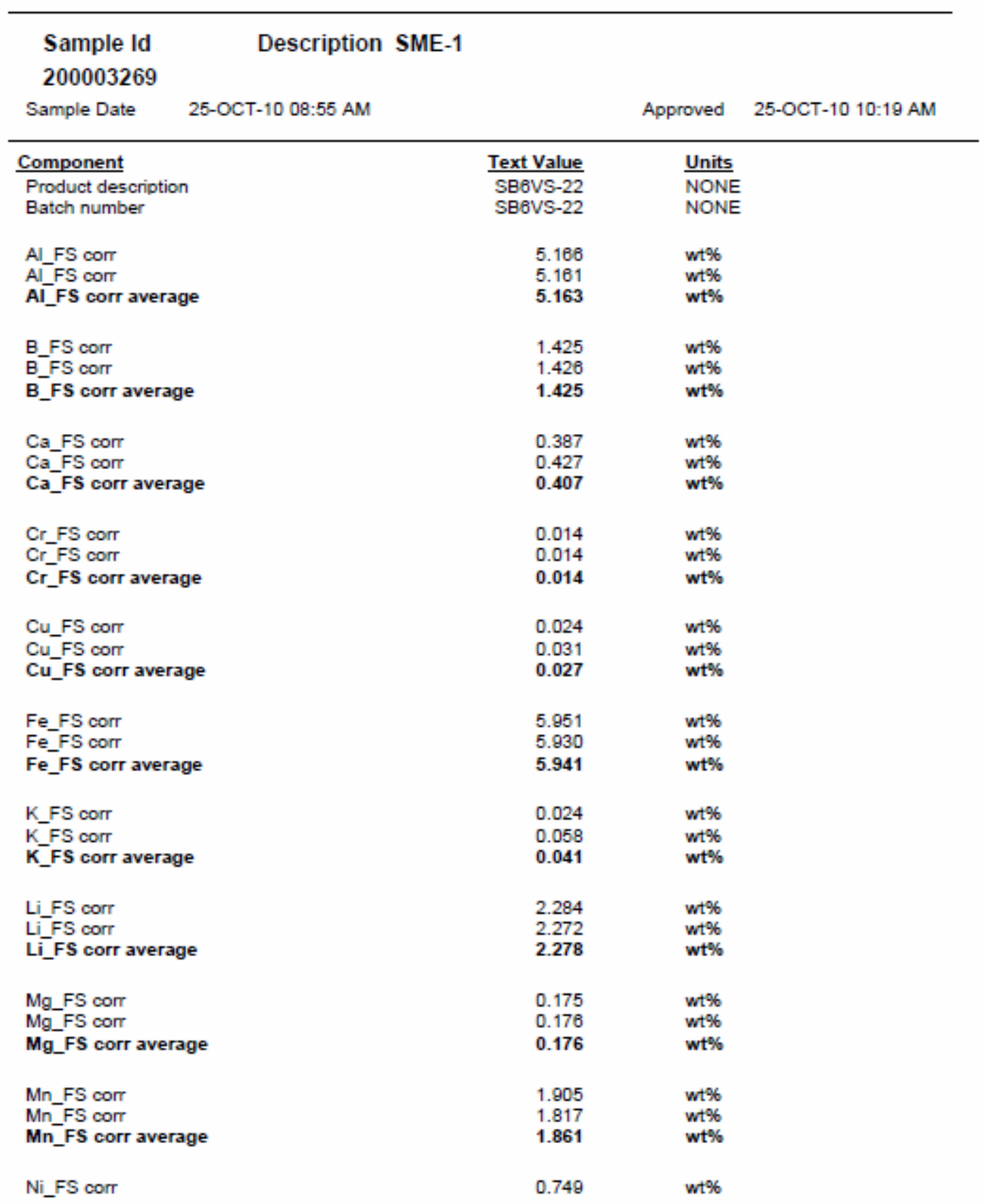


SRNL-STI-2011-00074

Revision 0

Table A4. DWPF Laboratory Measurements of SB6VS-21 (continued)

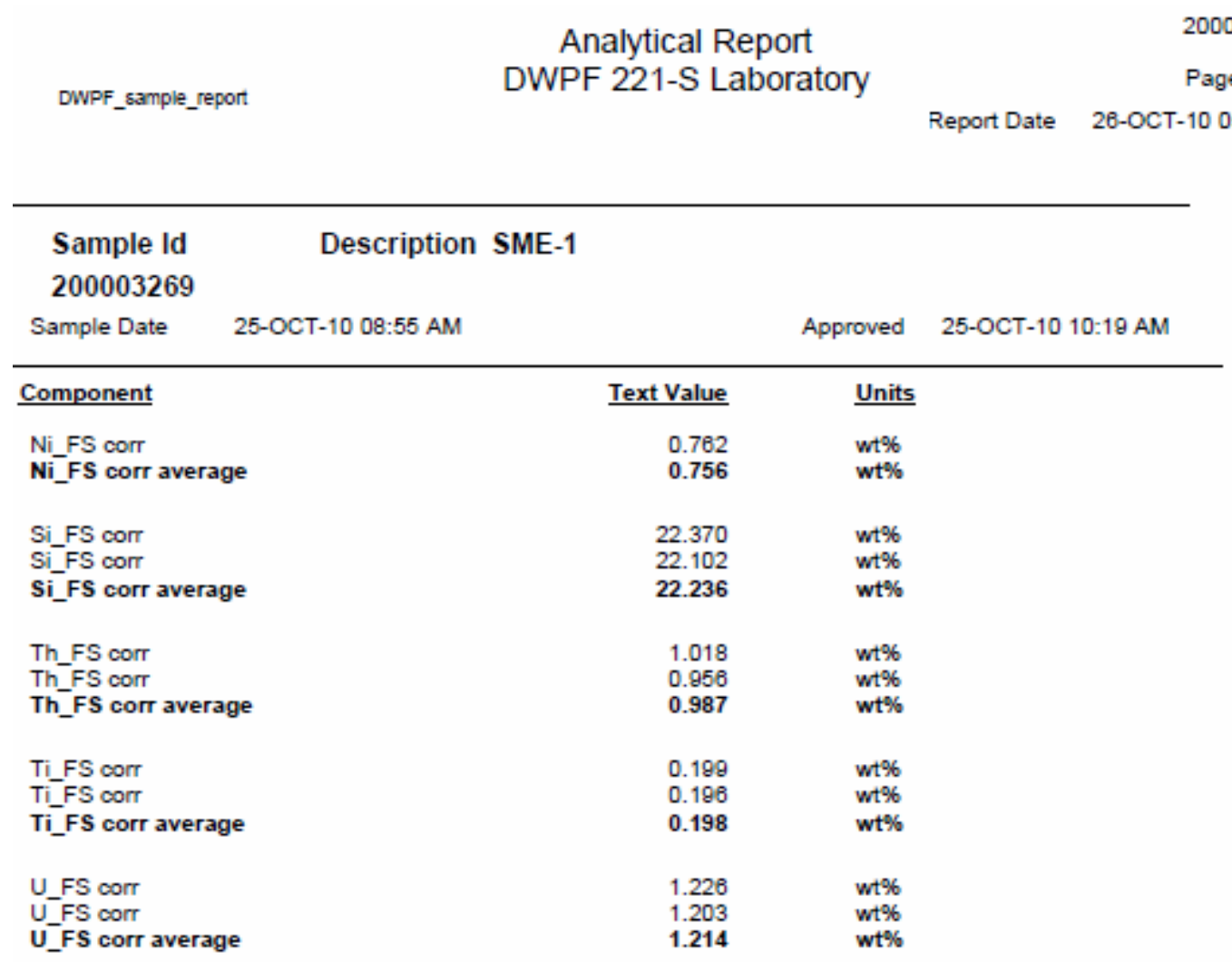


SRNL-STI-2011-00074

Revision 0

Table A5. DWPF Laboratory Measurements of SB6VS-22

Analytical Report

DWPF_sample_report
DWPF 221-S Laboratory
200003270

Page 1 of 2

Submission ID 100003270

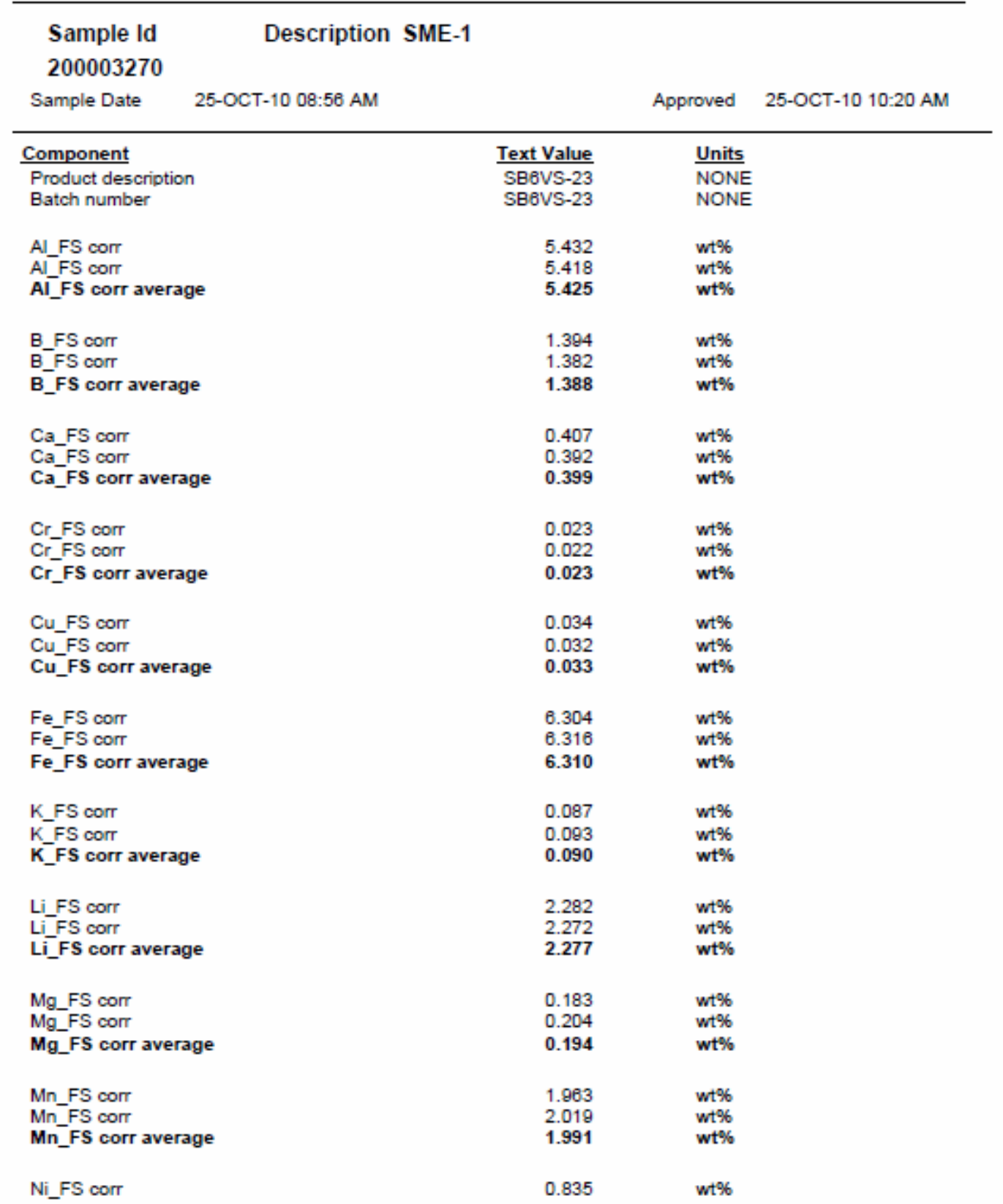


SRNL-STI-2011-00074

Revision 0

Table A5. DWPF Laboratory Measurements of SB6VS-22 (continued)

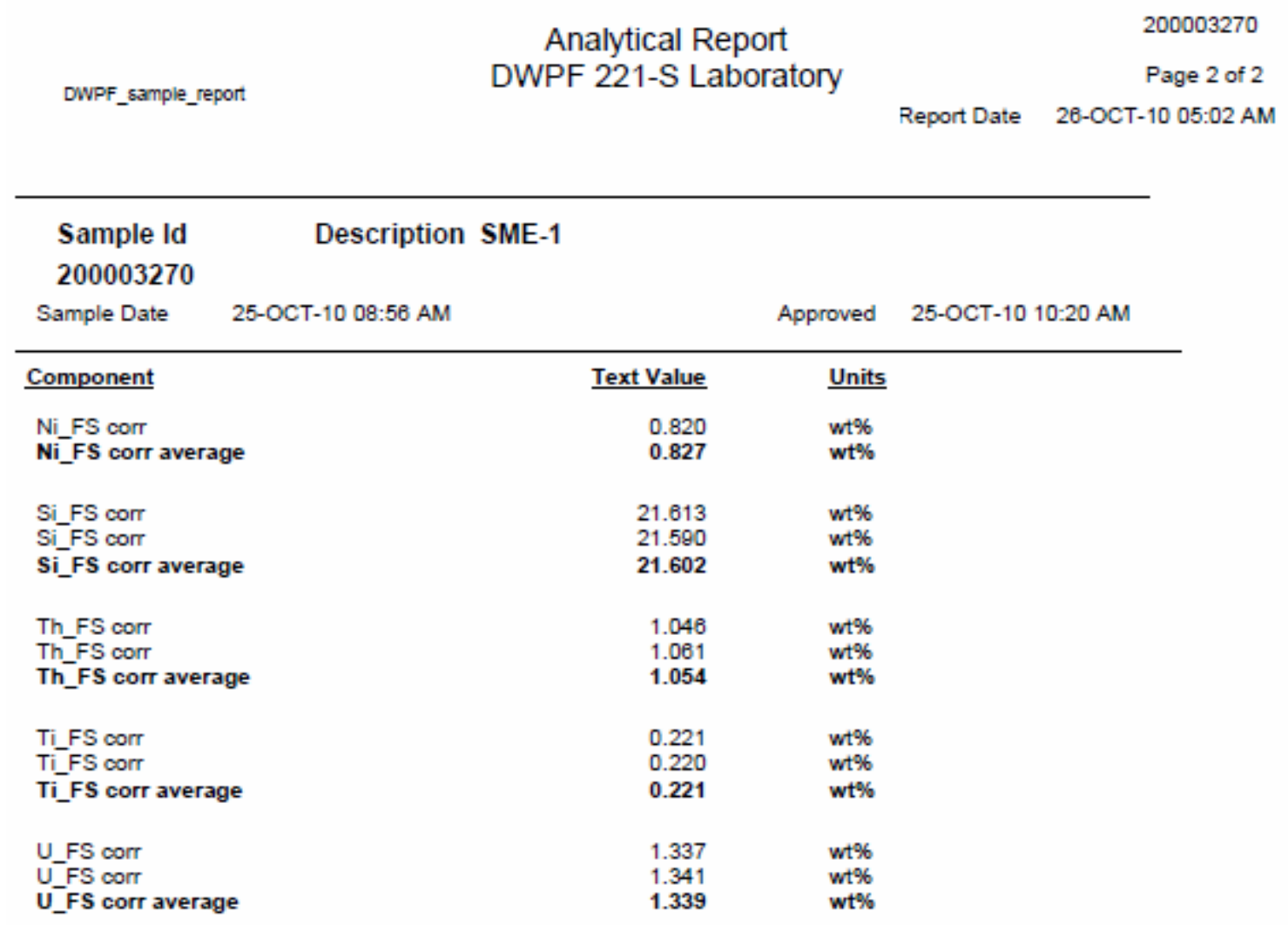


Exhibit A1. Comparison Plots by Oxide

Revision 0
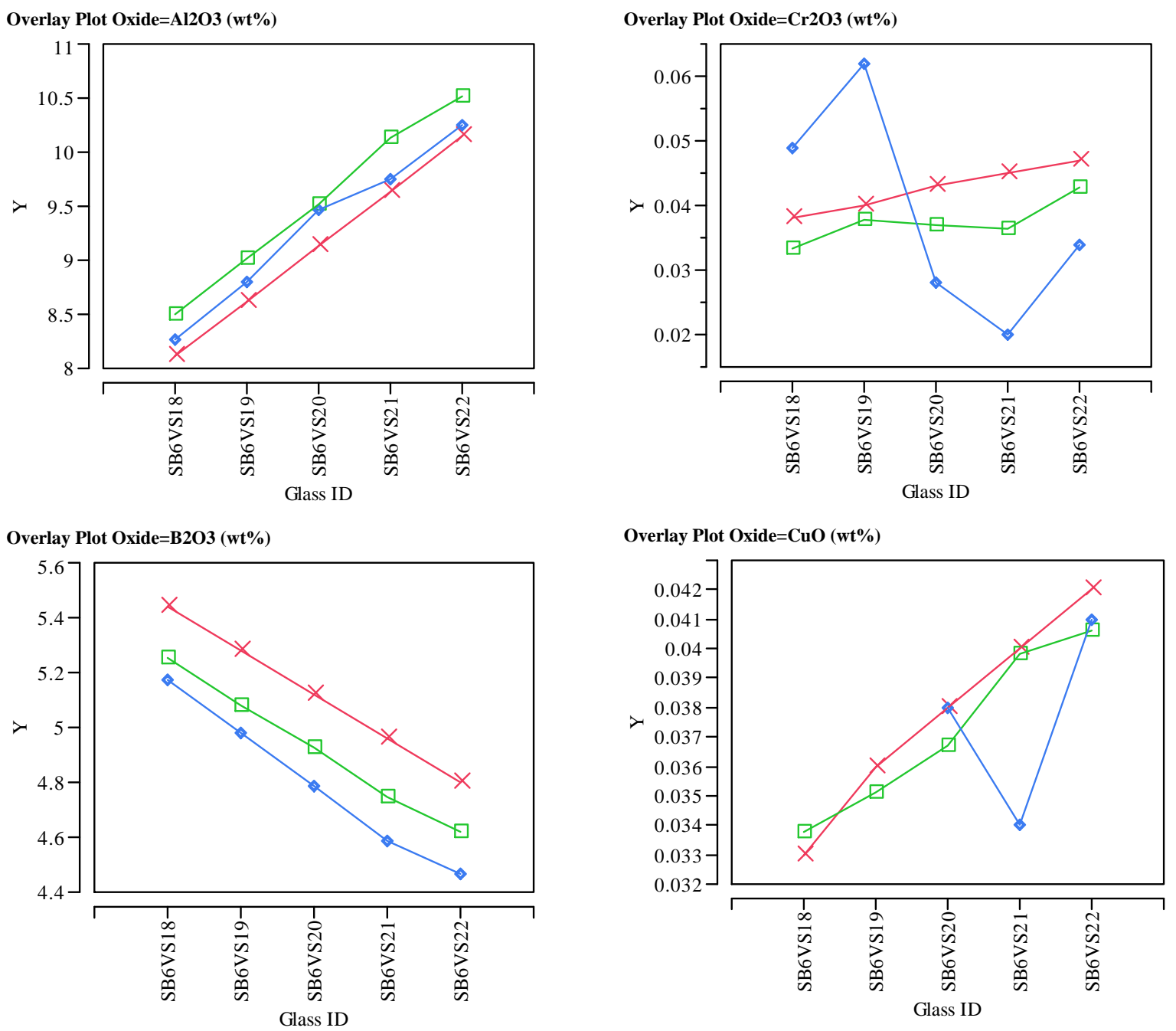

Overlay Plot Oxide $=\mathrm{CaO}(\mathrm{wt} \%)$

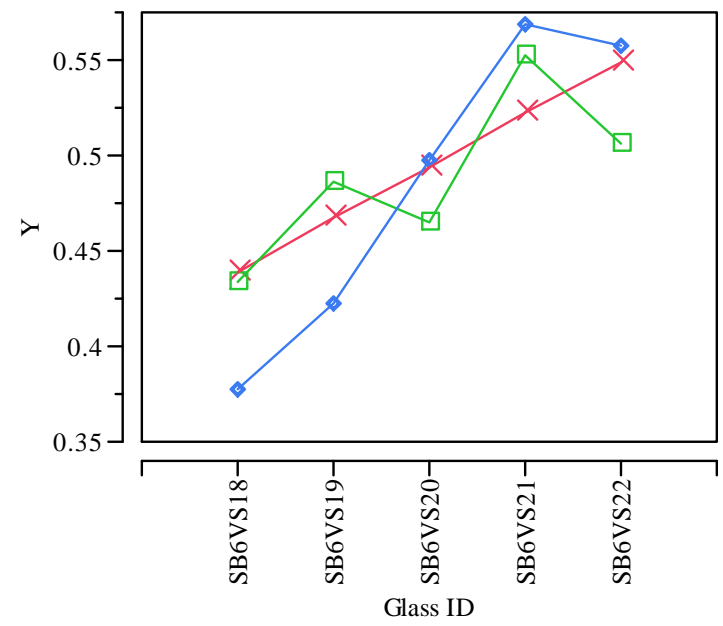

Overlay Plot Oxide=Fe2O3 (wt \%)

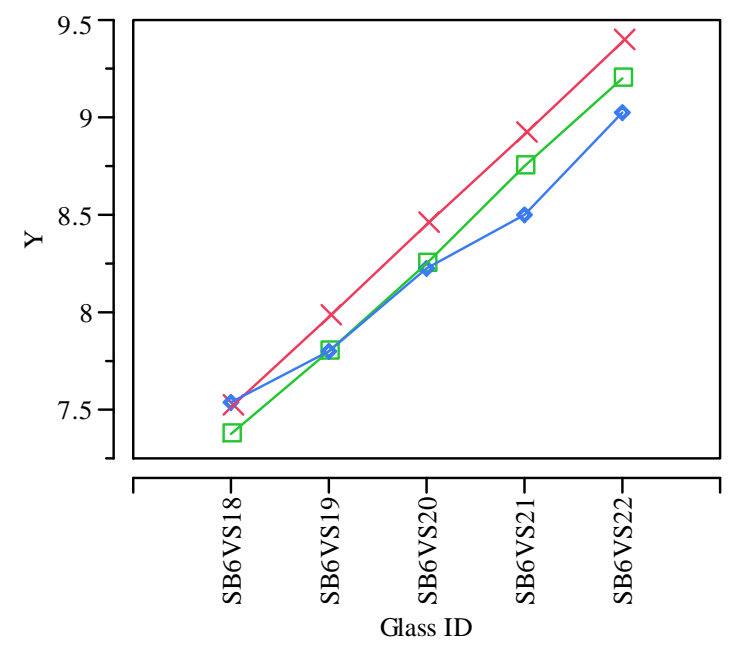

$\mathrm{Y}$

$x$ - Targeted wt $\%$

- - SRNL Measured wt $\%$

$\diamond$ DWPF Lab Measured wt\% 
Exhibit A1. Comparison Plots by Oxide

Revision 0

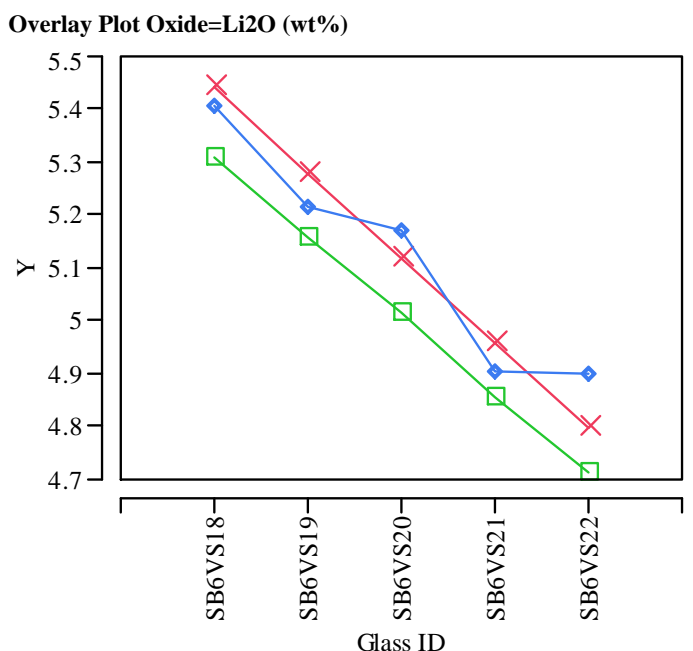

Overlay Plot Oxide=MgO (wt \%)

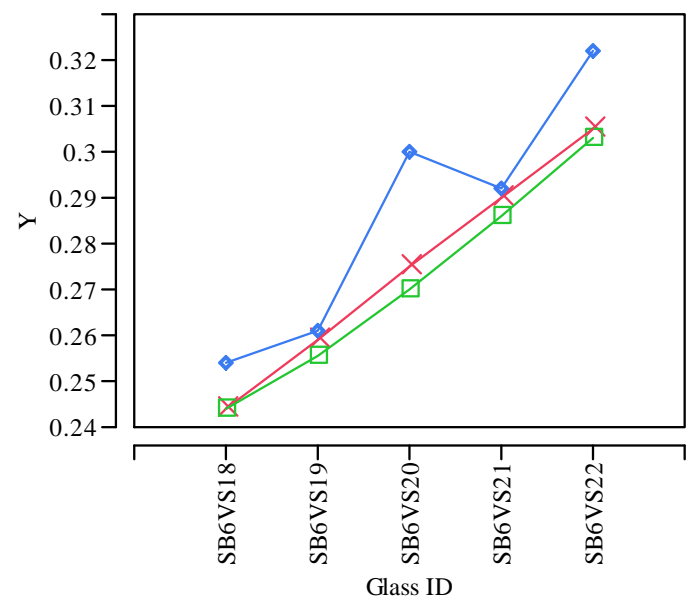

Overlay Plot Oxide=MnO (wt\%)

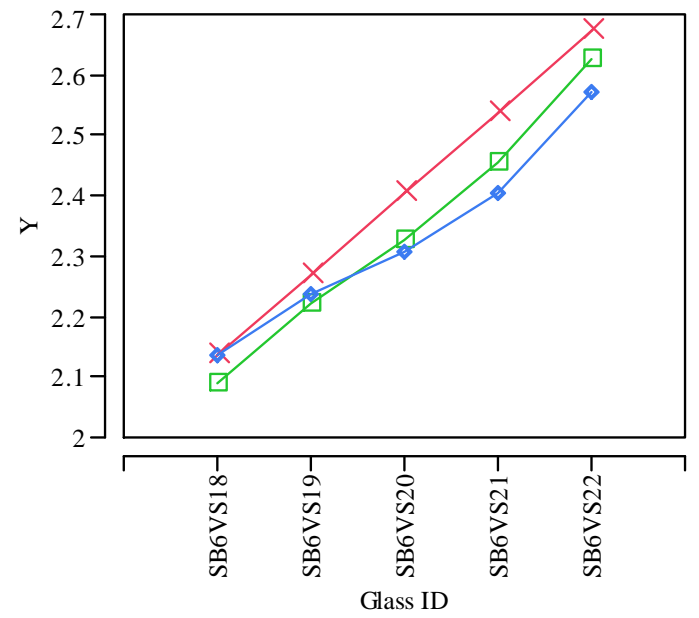

Y

$x$ - Targeted wt $\%$

口 - SRNL Measured wt \%

$\diamond$ DWPF Lab Measured wt $\%$
Overlay Plot Oxide=NiO (wt \%)

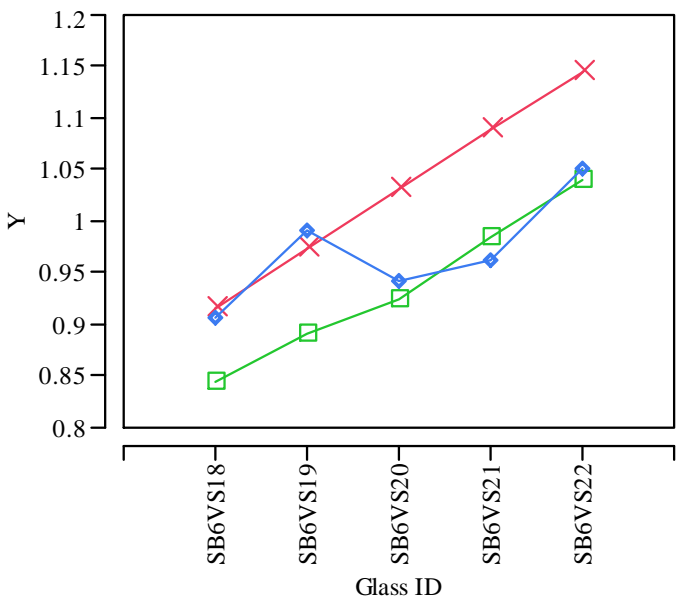

Overlay Plot Oxide=SiO2 (wt\%)

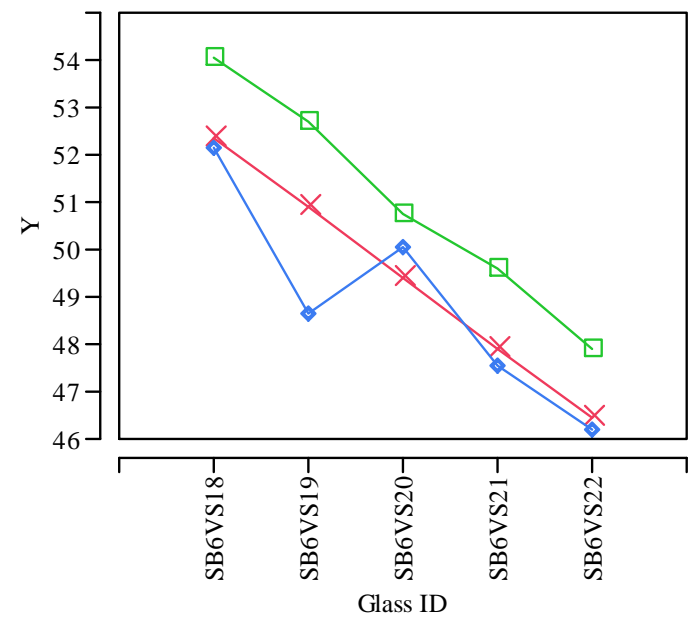

Overlay Plot Oxide=ThO2 (wt \%)

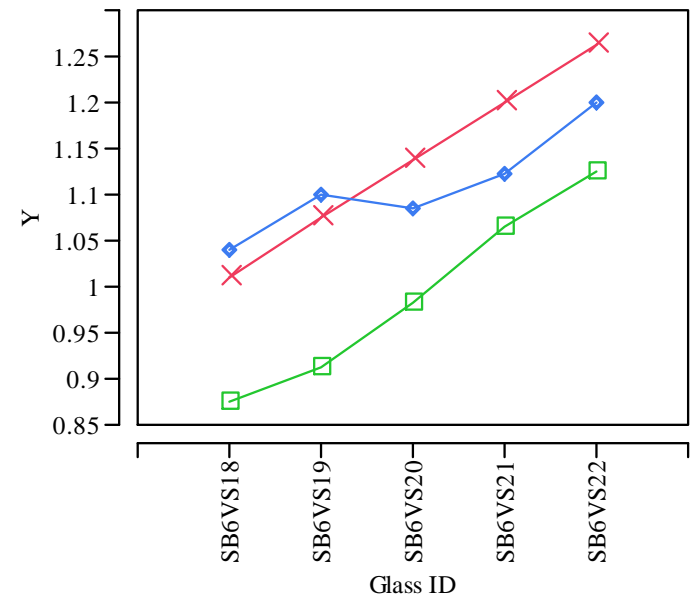

Y

$x$ - Targeted wt $\%$

a - SRNL Measured wt \%

• DWPF Lab Measured wt\% 
Exhibit A1. Comparison Plots by Oxide

Revision 0

Overlay Plot Oxide=TiO2 (wt\%)

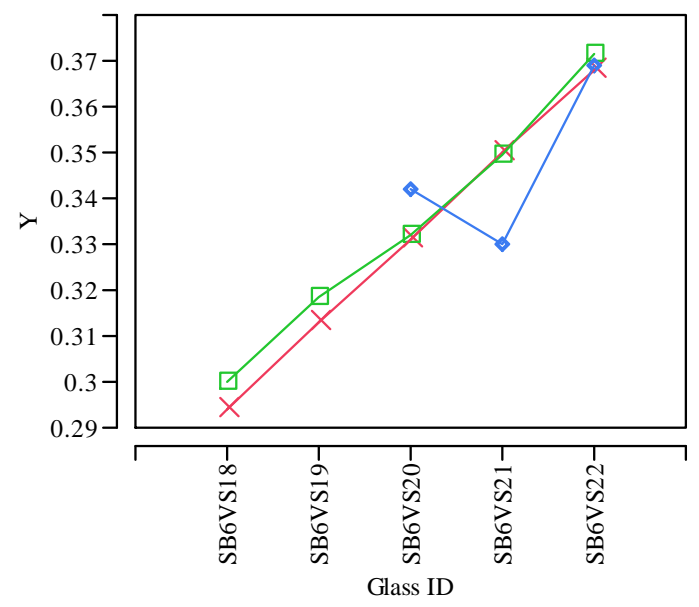

Overlay Plot Oxide=U308 (wt\%)

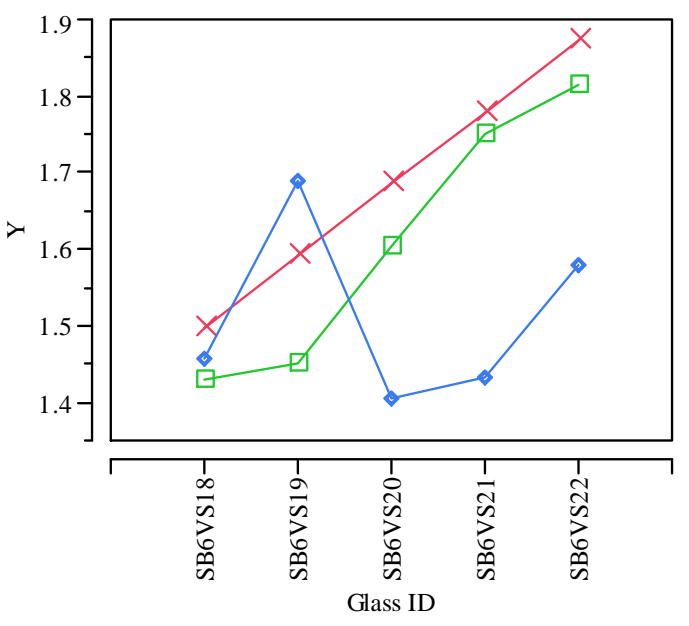

Y

x — Targeted wt $\%$

口- SRNL Measured wt $\%$

$\diamond$ DWPF Lab Measured wt $\%$ 


\section{Distribution:}

\begin{tabular}{|c|c|}
\hline Name: & Location: \\
\hline Sharon Marra & 773-A \\
\hline Connie Herman & 999-W \\
\hline Charles J. Coleman & 773-A \\
\hline Clint Gregory & 773-A \\
\hline Mark Barnes & 773-A \\
\hline Patricia Lee & $703-41 \mathrm{~A}$ \\
\hline Gene Shine & $703-41 \mathrm{~A}$ \\
\hline Damon R. Click & $773-\mathrm{A}$ \\
\hline L. Curtis Johnson & 773-A \\
\hline Michael Stone & 999-W \\
\hline John Pareizs & 773-A \\
\hline David Peeler & 999-W \\
\hline Tommy Edwards & 999-W \\
\hline Kevin Fox & 999-W \\
\hline Fabienne Johnson & 999-W \\
\hline Charles Crawford & $773-42 \mathrm{~A}$ \\
\hline David Best & 999-W \\
\hline John Occhipinti & 704-S \\
\hline Jonathan Bricker & $704-27 S$ \\
\hline John Iaukea & 704-30S \\
\hline Aaron Staub & $704-27 S$ \\
\hline Jeff Ray & 704-S \\
\hline Robert Hinds & 704-S \\
\hline Terri Fellinger & $704-26 S$ \\
\hline Ryan McNew & 704-S \\
\hline Michael T. Hart & $210-S$ \\
\hline Roger N. Mahannah & $704-28 S$ \\
\hline Michael T. Feller & $704-28 S$ \\
\hline Omar Cardona-Quiles & $704-24 S$ \\
\hline Amanda Shafer & $704-27 S$ \\
\hline Mason Clark & $704-27 S$ \\
\hline Helen Pittman & $704-27 \mathrm{~S}$ \\
\hline Hank Elder & $704-24 S$ \\
\hline Bill Holtzscheiter & $704-15 S$ \\
\hline Pat Vaughan & $773-41 \mathrm{~A}$ \\
\hline
\end{tabular}

